\title{
Survey of Microinvasive Glaucoma Surgery and Other Glaucoma Surgical Experience among United States Ophthalmology Residency Programs
}

\author{
Kevin M. Halenda, MD ${ }^{1}$ Tae Jin Lee, $\mathrm{PhD}^{2}$ Ashok Sharma, $\mathrm{PhD}^{2}$ Amy J. Estes, MD ${ }^{1}$ \\ Kathryn E. Bollinger, MD PhD ${ }^{1}$ \\ ${ }^{1}$ Department of Ophthalmology, Medical College of Georgia at \\ Augusta University, Augusta, Georgia \\ 2 Department of Population Health Science, Medical College of \\ Georgia at Augusta University, Augusta, Georgia, \\ Address for correspondence Kathryn E. Bollinger, MD, PhD, 1120 \\ 15th St., BA-2320, Augusta, GA 30912 \\ (e-mail: kbollinger@augusta.edu). \\ J Acad Ophthalmol 2021;13:e108-e113.
}

\begin{abstract}
Keywords

- glaucoma

- microinvasive glaucoma surgery

- residents

- surgical experience

Purpose The aim of the study is to assess the state of glaucoma surgical training in United States ophthalmology residency programs, including experience with microinvasive glaucoma surgery (MIGS).

Design The design of the study is anonymous, internet-based national survey. Participants Current United States ophthalmology residents of residency programs accredited by the Accreditation Council for Graduate Medical Education (ACGME).

Methods An anonymous survey link was emailed to all 120 accredited United States ophthalmology residency programs inviting residents to participate in an assessment of residency glaucoma surgical experience. Survey responses were collected between January 21, 2019 and March 4, 2019 and analyzed using descriptive statistics.

Main Outcome Measures The main outcomes of the study are demographic information, practice intentions, and anticipated primary surgical experience with ACGME-required glaucoma procedures and MICS procedures, as self-reported by U.S. ophthalmology residents. Results Of the estimated 1,479 U.S. ophthalmology residents, 161 residents participated (10.9\%). A total of 118 residents (73.2\%) reported any degree of anticipated MIGS primary surgical experience during residency, with the iStent being the most familiar technique. The likelihood of any anticipated MIGS experience during residency was not significantly different by geographic region ( $p=0.16$ ), however, anticipated volume varied significantly $(p=0.037)$. Of the 113 respondents who reported an intention to manage glaucoma surgically in their eventual practice, $25(22.1 \%)$ reported no anticipated primary MIGS experience during residency. $73.3 \%$ of residents anticipating MIGS experience anticipated 0 to 10 cases, with $42.9 \%$ anticipating less than 5 cases as primary surgeon.

Conclusion MIGs are not a required component of the glaucoma surgical curriculum for U.S. ophthalmology residents. Although the majority of ophthalmology residents surveyed intend to manage glaucoma surgically in eventual practice, most receive minimal experience with these novel techniques during residency. Surgical training is variable by geographic region.
\end{abstract}

received

July 23, 2020 accepted after revision September 22, 2020
DOI https://doi.org/ 10.1055/s-0040-1721072. ISSN 2475-4757.

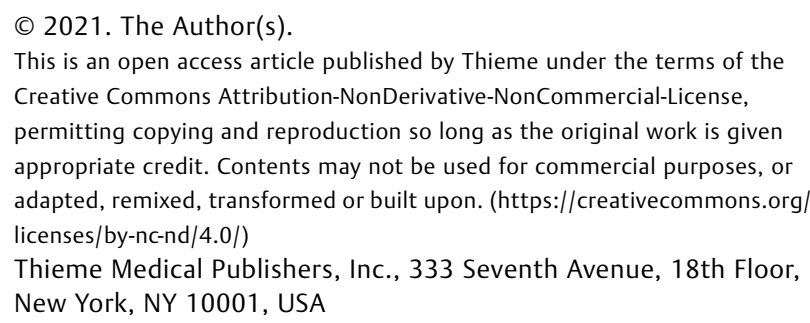

This is an open access article published by Thieme under the terms of the Creative Commons Attribution-NonDerivative-NonCommercial-License, permitting copying and reproduction so long as the original work is given appropriate credit. Contents may not be used for commercial purposes, or adapted, remixed, transformed or built upon. (https://creativecommons.org/ licenses/by-nc-nd/4.0/)

Thieme Medical Publishers, Inc., 333 Seventh Avenue, 18th Floor, New York, NY 10001, USA 
Glaucoma is a leading cause of blindness and ocular morbidity globally and is projected to afflict over 110 million people worldwide by $2040 .{ }^{1}$ Reduction of intraocular pressure (IOP), which can be accomplished through a variety of medications, laser therapies, and surgical interventions, is currently the only established glaucoma treatment. ${ }^{2}$ Incisional surgical interventions are indicated when more conservative measures fail to produce sufficient IOP reduction and in cases of patient adherence challenges. $^{3}$

The emergence of microinvasive/minimally invasive glaucoma surgery (MIGS), has altered the modern surgical approach to the management of primary open angle glaucoma. While generally less disruptive to the ocular tissues albeit with more modest IOP-lowering efficacy, ${ }^{4}$ MIGS procedures enjoy increasing popularity and recognition by the American Glaucoma Society as a useful addition to the glaucoma surgical armamentarium. ${ }^{5}$ Nevertheless, there is currently no standardized required experience with these procedures during ophthalmology residency training in the United States. The requirement for glaucoma procedural experience put forth by the Accreditation Council for Graduate Medical Education (ACGME) is for an ophthalmology resident to achieve a minimum of five Filtering and/or Shunting procedures, five laser trabeculoplasties, and four laser iridotomies during training, a standard most recently updated in $2013 .{ }^{6}$ The degree to which U.S. ophthalmology residents are exposed to MIGS techniques during their surgical training, and how this may affect future practice patterns and delivery of glaucoma care is presently unknown. The purpose of this study is to survey current U.S. ophthalmology residents regarding their experience with surgical management of glaucoma, including MIGS techniques, and eventual practice intentions.

\section{Methods}

The study protocol was submitted to the Augusta University Institutional Review Board and was deemed exempt from formal review. A 10-question internet-based questionnaire was subsequently emailed to all 120 accredited U.S. ophthalmology residency programs listed on the ACGME website at the time of study design. The survey instrument was designed and utilized to collect responses anonymously through Qualtrics software (Qualtrics Inc, Provo, UT). The email solicitation containing the survey link was emailed to both the program directors and program coordinators when their contact information was available, requesting subsequent distribution to residents in their respective programs. The questionnaire contained questions surveying current residents regarding their year in training, geographic area of residency program, intended practice setting, intended subspecialty, intended style of management of glaucoma post training, expected surgical volume of both ACGME-required glaucoma procedures (filtering/shunting procedures, laser trabeculoplasty, laser iridotomy), and MIGS procedures, as well as anticipated experience with specific MIGS techniques as primary surgeon (-Supplementary Fig. S1). The survey stratified anticipated primary surgical experience in four categories: $<5,5$ to 10,10 to 20 , or $>20$ procedures. The survey remained open collecting responses between January 21, 2019 and March 4, 2019. Reminder emails to residency programs were sent at 2-week intervals during this time period. Collected survey responses were evaluated using chi-square analysis.

\section{Results}

A total of 161 survey responses were collected. As ascertained from the ACGME website, based on a total of 1,479 resident positions, this amounted to a $10.9 \%$ response rate nationally. Demographics of survey respondents are reported in - Table 1 . $44.1 \%$ of respondents were third year residents, $37.3 \%$ were second year residents, and $18.6 \%$ were first year residents. The most common intended subspecialty reported by

Table 1 Demographics of survey respondents

\begin{tabular}{|l|l|l|}
\hline & Number & Percentage \\
\hline Total US residents & 1,479 & \\
\hline Total respondents & 161 & $10.9 \%$ \\
\hline Year of training & & \\
\hline 1 & 30 & $18.6 \%$ \\
\hline 2 & 60 & $37.3 \%$ \\
\hline 3 & 71 & $44.1 \%$ \\
\hline Intended specialty & & \\
\hline General/Comprehensive & 61 & $37.9 \%$ \\
\hline Cornea/Refractive & 22 & $13.7 \%$ \\
\hline Glaucoma & 31 & $19.3 \%$ \\
\hline Oculoplastics & 8 & $5.0 \%$ \\
\hline Pediatrics/Strabismus & 8 & $5.0 \%$ \\
\hline Neuro-ophthalmology & 1 & $0.6 \%$ \\
\hline Retina (surgical) & 25 & $15.5 \%$ \\
\hline Retina (medical) & 2 & $1.2 \%$ \\
\hline Uveitis & 3 & $1.9 \%$ \\
\hline Intended practice setting & & \\
\hline Academics & 40 & $24.8 \%$ \\
\hline Private practice-solo & 5 & $3.1 \%$ \\
\hline Private practice-group & 102 & $63.3 \%$ \\
\hline Employee & 14 & $8.7 \%$ \\
\hline Geographic region & & \\
\hline Northeast & 29 & $18.0 \%$ \\
\hline Southeast & 47 & $29.2 \%$ \\
\hline Southwest & 23 & $14.3 \%$ \\
\hline Midwest & 43 & $26.7 \%$ \\
\hline West & 19 & $11.8 \%$ \\
\hline Intended glaucoma management & & \\
\hline None & 25 & $15.5 \%$ \\
\hline Medical only & 23 & $14.3 \%$ \\
\hline Minimal surgical & 76 & $47.2 \%$ \\
\hline Advanced surgical & $23.0 \%$ \\
\hline
\end{tabular}


respondents was comprehensive (37.9\%) followed by glaucoma (19.3\%). Most survey respondents reported an intention to practice in a group private practice setting (63\%) upon completion of residency training. Residents from all geographic regions were represented, with the Southeast being the most common (29.2\%) and the West being the least common (11.8\%) source of respondents, respectively. Concerning management of glaucoma patients, a majority of respondents (70.2\%) reported an intention to manage glaucoma surgically upon completion of training. Among these respondents, 47.2\% reported an intention to manage glaucoma with less invasive techniques (e.g., laser trabeculoplasty, phacoemulsification, MIGS), while $23.0 \%$ reported an intention to include more advanced glaucoma procedures (glaucoma drainage device implants and trabeculectomies) in their eventual practice.

Overall, $73.3 \%$ of survey respondents reported actual or anticipated primary surgical experience with any of the MIGS techniques referenced in the survey item (-Table 2 ). The most common technique with which residents reported experience was the iStent (Glaukos, San Clemente, CA), at $57.8 \%$. The second most common reported MIGS technique was the Kahook Dual Blade goniotomy (New World Medical, Rancho Cucamonga, CA). Gonioscopy-assisted transluminal trabeculotomy and the XEN Gel Stent (Allergan, Dublin, Ireland) were the only other MIGS techniques with which at least $10 \%$ of U.S. ophthalmology residents reported actual or anticipated experience (-Table 2 ). Regarding cyclodestructive procedures, $75.8 \%$ and $24.8 \%$ reported actual or anticipated experience with transscleral cyclophotocoagulation and endocyclophotocoagulation, respectively (-Table 2). Among traditional glaucoma surgical techniques, glaucoma drainage device implantation was more commonly reported to be a component of U.S. ophthalmology residency training (85.1\%) than trabeculectomy (60.2.\%) (-Table 2).

The anticipated volume performed as primary surgeon during residency of the four different categories of glaucoma procedures considered (aqueous shunt/trabeculectomy, MIGS, iridotomy, trabeculoplasty) was significantly different $(p<0.0001)$ ( - Fig. 1). The majority of respondents $(73.3 \%)$ anticipated performing 0 to 10 MIGS cases during residency. The most common anticipated primary MIGS volume performed during residency was $<5$ (42.9\%), followed by 5 to 10 (30.4\%). The majority of respondents (50.3\%) anticipated 5 to 10 tube shunts/trabeculectomies during residency training. Comparatively, the anticipated volume categories of trabeculoplasties and iridotomies performed during residency was more evenly distributed among higher volume categories (-Fig. $\mathbf{1}$ ).

Upon analysis of MIGS procedure volume by geographic region, a significant difference was demonstrated $(p=0.037)$. Anticipated iridotomy volume by geographic region also reached statistical significance in our analysis $(p=0.049)$. However, no significant difference was identified in the volume of aqueous tube shunts/trabeculectomies $(p=0.17)$ or trabeculoplasties anticipated by geographic region ( $p=0.42$ ). Additionally, intended glaucoma management style was also not statistically different among the different geographic regions ( $p=0.19)$ (-Fig. 2).
Table 2 Anticipated primary glaucoma surgical procedure experience

\begin{tabular}{|c|c|c|}
\hline & $\begin{array}{l}\text { No. of } \\
\text { Respondents }\end{array}$ & $\begin{array}{l}\text { \% Respondents } \\
\text { anticipating } \\
\text { primary surgical } \\
\text { experience }\end{array}$ \\
\hline \multicolumn{3}{|l|}{ Cataract surgery } \\
\hline Phacoemulsification & 156 & $96.9 \%$ \\
\hline \multicolumn{3}{|l|}{ Shunting/filtering procedures } \\
\hline Trabeculectomy & 97 & $60.2 \%$ \\
\hline Glaucoma drainage implant & 137 & $85.1 \%$ \\
\hline \multicolumn{3}{|l|}{ Laser procedures } \\
\hline Laser Iridotomy & 153 & $95.0 \%$ \\
\hline Laser trabeculoplasty & 156 & $97.0 \%$ \\
\hline \multicolumn{3}{|l|}{ Cyclodestructive procedures } \\
\hline $\begin{array}{l}\text { Transscleral } \\
\text { cyclophotocoagulation }\end{array}$ & 122 & $75.8 \%$ \\
\hline $\begin{array}{l}\text { Endocyclophotocoagulation } \\
\text { (ECP) }\end{array}$ & 40 & $24.8 \%$ \\
\hline \multicolumn{3}{|l|}{ MIGS procedures } \\
\hline iStent & 93 & $57.8 \%$ \\
\hline iStent SUPRA & 10 & $6.2 \%$ \\
\hline CyPass & 13 & $8.1 \%$ \\
\hline Kahook dual blade & 56 & $34.8 \%$ \\
\hline $\begin{array}{l}\text { Gonioscopy-assisted } \\
\text { transluminal } \\
\text { trabeculotomy (GATT) }\end{array}$ & 26 & $16.1 \%$ \\
\hline Trabectome & 13 & $8.1 \%$ \\
\hline TRAB 360 & 12 & $7.5 \%$ \\
\hline Ab interno canaloplasty & 8 & $5.0 \%$ \\
\hline VISCO360 & 4 & $2.5 \%$ \\
\hline Hydrus Microstent & 9 & $5.6 \%$ \\
\hline XEN gel stent & 23 & $14.8 \%$ \\
\hline InnFocus Microshunt & 0 & $0.0 \%$ \\
\hline Any MIGS & 118 & $73.3 \%$ \\
\hline Any MIGS/ECP & 123 & $76.4 \%$ \\
\hline
\end{tabular}

Abbreviation: MIGS, microinvasive glaucoma surgery.

As respondents' anticipated MIGS primary surgical volume was seen to vary significantly by geographic region, we performed additional sub-analyses dividing respondents into those with any degree of anticipated MIGS experience during residency, and those with none. Although anticipated MIGS volume varied significantly by geographic region, the likelihood of any MIGS exposure during residency did not vary significantly across geographic region $(p=0.16)$. Intended glaucoma management style was also not found to differ significantly between residents anticipating any MIGS experience or none $(p=0.085)(-$ Fig. 3 ). Of the 113 respondents who reported an intention to manage glaucoma surgically to any degree in their practice, 25 (22\%) anticipated no MIGS experience as primary surgeon during their residency training. 


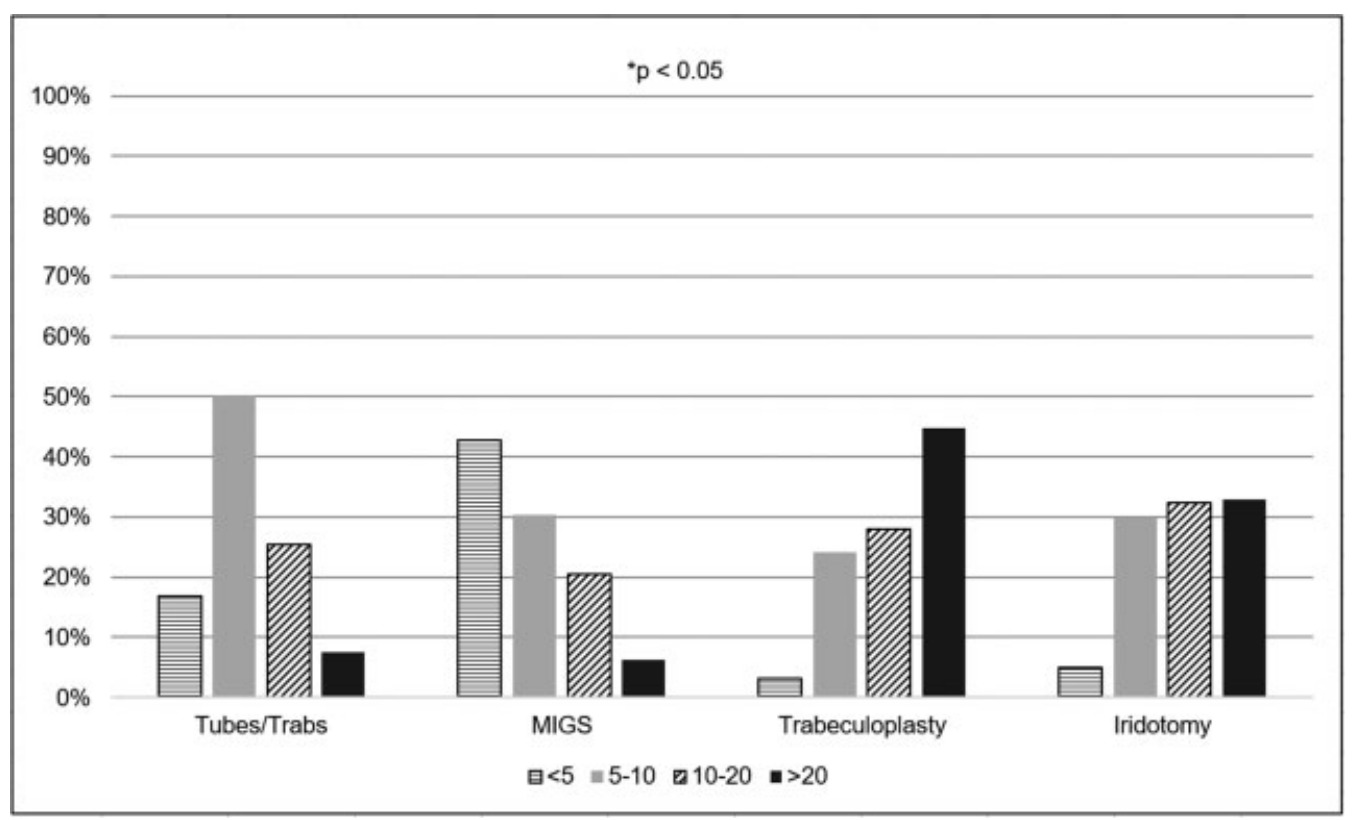

Fig. 1 Anticipated primary surgical volume by glaucoma procedure.

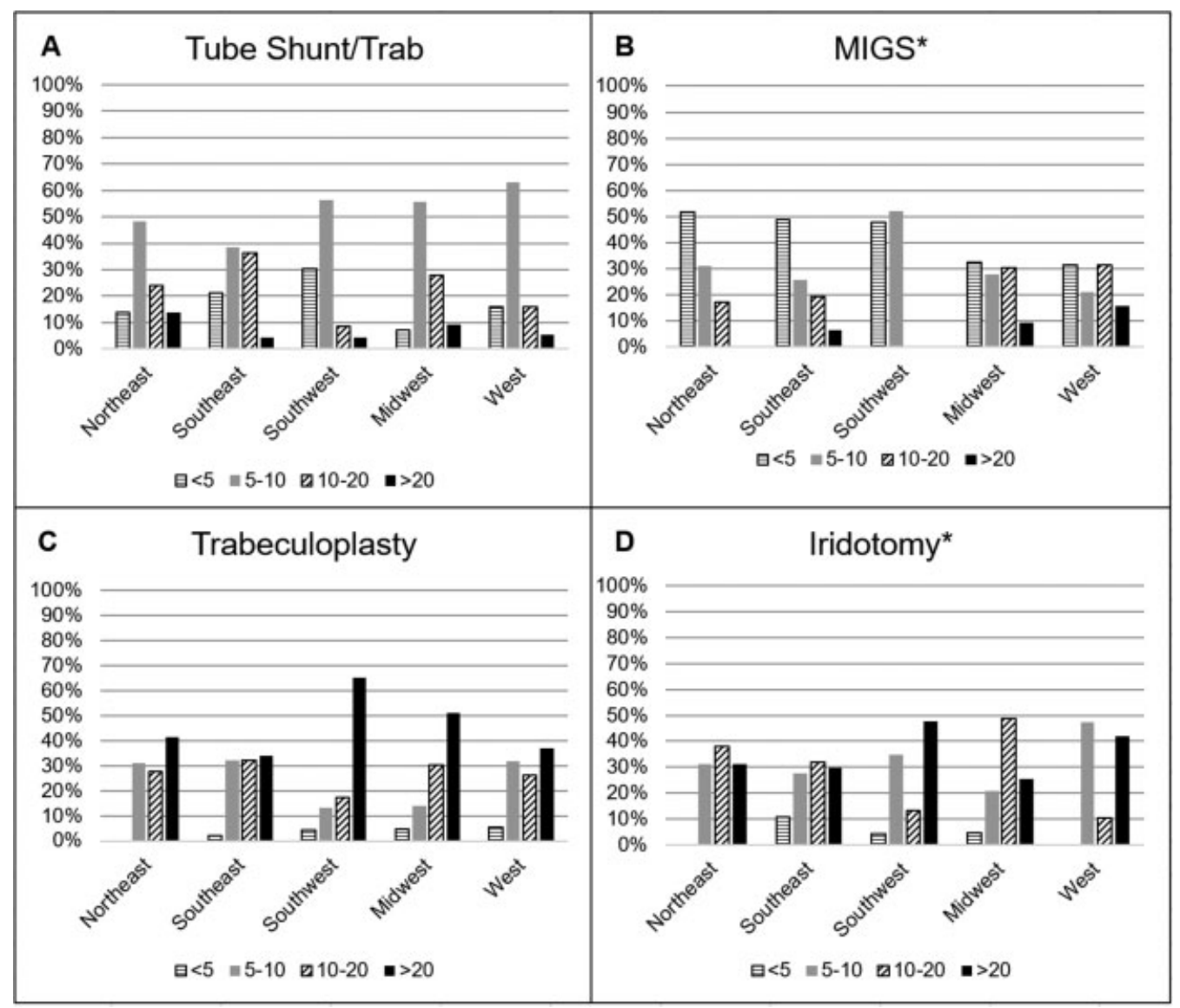

Fig. 2 Anticipated primary glaucoma surgical volume by geographic region. ${ }^{*} p<0.05$.

\section{Discussion}

Although MIGS techniques are increasingly popular in the surgical paradigm of glaucoma due to perceived safety and recovery benefits compared with traditional glaucoma sur- gical techniques, ${ }^{7}$ these methods are not currently a standard required component of ophthalmology residency training in the United States. To our knowledge, no previous study has specifically investigated the state of MIGS training during residency. Our national survey sample suggests that while 


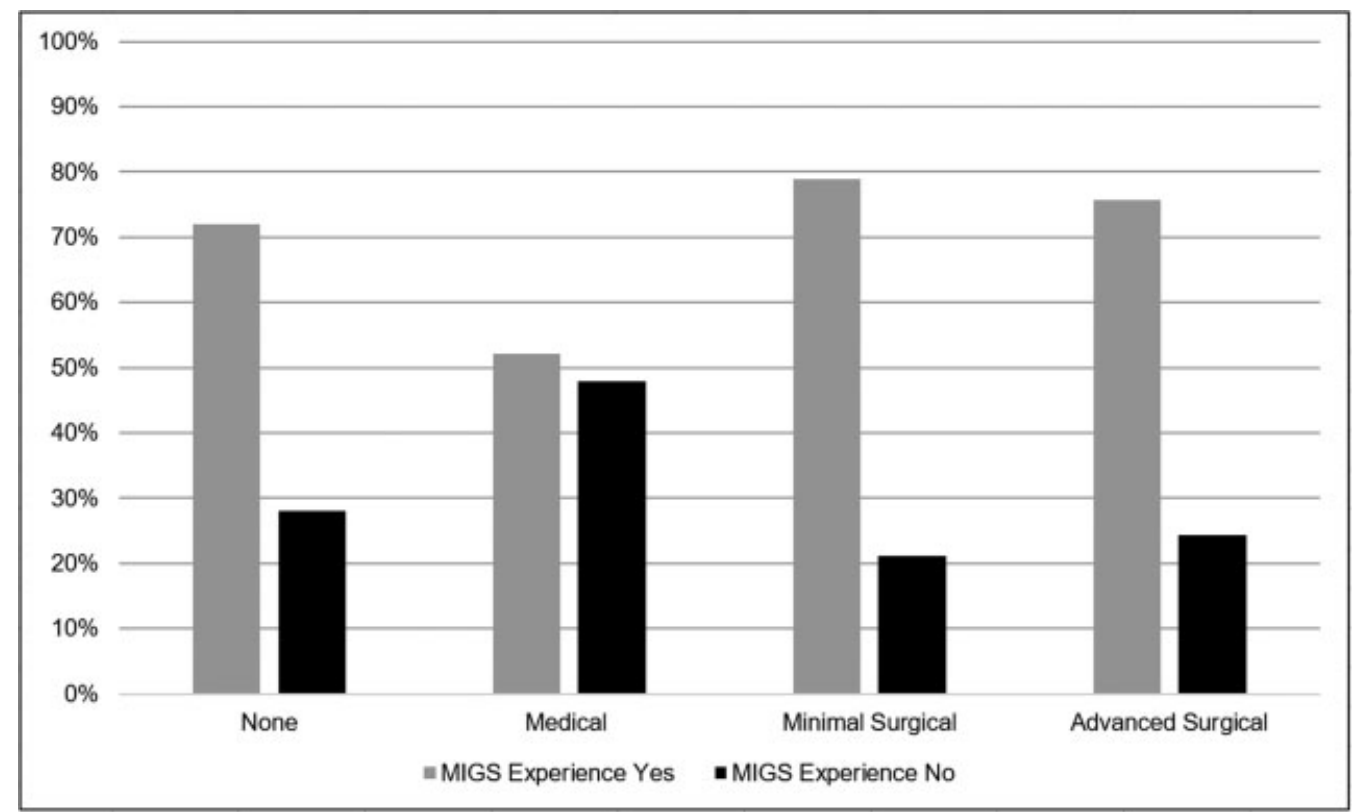

Fig. 3 Any anticipated MIGS experience by anticipated glaucoma practice. MIGS, microinvasive glaucoma surgery.

the majority of ophthalmologists in training in the United States intend to utilize minimally invasive surgical techniques to manage glaucoma in their practice, including MIGS, a considerable portion of such trainees may receive little MIGS exposure during their residencies. In addition, we have observed significant variation in the volume of anticipated MIGS procedures performed by geographic region. The reasons underlying this geographic variability are unclear at this time and warrant further future investigation.

Studies examining ACGME resident glaucoma case logs suggest that national glaucoma surgical trends are also reflected at the level of residency training experience. ${ }^{8,9}$ Echoing national trends of declining use of the trabeculectomy and increasing usage of the aqueous tube shunt, ${ }^{8}$ examination of ACGME data collected between 2009 to 2016 has shown a $20 \%$ decline in primary filtering surgery volume, and a parallel $40 \%$ increase in glaucoma tube shunt implantation. ${ }^{9}$ Chadha et al reported that MIGS were not specifically tracked by the ACGME case logs at the time of their study, nor were all MIGS procedures tracked in the Association of University Professors of Ophthalmology glaucoma fellow case logs at that time. ${ }^{9}$ Presently, it appears that common MIGS current procedural terminology codes are tracked by the ACGME case log system under the general categories of "shunting" or "filtering" procedures. The most current (2018-2019) Ophthalmology National Resident Report released by the ACGME also provides incisional glaucoma surgery resident averages and percentiles only under these same general categories of "filtering procedures," "shunting procedures," and "other glaucoma procedures," without further subdivision into specific procedures or techniques utilized. ${ }^{10}$ Thus, our survey provides unique insight regarding the actual experience and exposure to these procedures in training programs.

The question of whether MIGS should be a component of the required primary glaucoma surgical curriculum for U.S. ophthalmology residents is a matter that should be weighed by surgical educators and policymakers. Because trainees are performing fewer trabeculectomies during residency, ${ }^{9}$ it may be reasonable to supplement this experience with an additional skillset. Nonetheless, MIGS procedures are uniquely difficult in certain respects, for example requiring the mastery of simultaneous gonioscopy with the nondominant hand to adequately visualize the target tissues. ${ }^{5}$ While residents have been shown to be capable of safely performing MIGS procedures, ${ }^{11}$ ophthalmology is a microsurgical field in which an estimated $9 \%$ of residents may struggle to develop technical competency, ${ }^{12}$ and it can be argued that MIGS may be more appropriately learned during advanced training or adopted later on in a surgeon's career.

Our study faces several limitations. A national response rate of approximately $11 \%$ may somewhat limit the generalizability of our conclusions, although our study sample demonstrated a relatively even representation of geographic regions and resident class years. Additionally, a wide array of subspecialty interests was represented, without apparent disproportionate interest in specializing in glaucoma among participating trainees. Anticipated surgical volume was based on self-reported data and was also stratified into four categories to simplify survey participation. This approach however, appears to be valid, as $50 \%$ of our respondents reported an estimated 5 to 10 shunting and/or filtering procedures as primary surgeon during residency, which is similar to the ACGME national case log 50th percentile published for both of these procedural categories (5 [50\% percentile for these procedure categories]). ${ }^{10}$

\section{Disclosures}

The authors report that these data were presented in part as a poster at the 2020 meeting of the American Glaucoma Society on February 27, 2020. 
Funding

The authors report that this was an unfunded study.

\section{Conflict of Interest}

No conflicting relationship exists for any author.

\section{References}

1 Tham YC, Li X, Wong TY, Quigley HA, Aung T, Cheng CY. Global prevalence of glaucoma and projections of glaucoma burden through 2040: a systematic review and meta-analysis. Ophthalmology 2014;121(11):2081-2090

2 Weinreb RN, Aung T, Medeiros FA. The pathophysiology and treatment of glaucoma: a review.JAMA 2014;311(18):1901-1911

3 Sharaawy T, Bhartiya S. Surgical management of glaucoma: evolving paradigms. Indian J Ophthalmol 2011;59(suppl): S123-S130

4 Saheb H, Ahmed II. Micro-invasive glaucoma surgery: current perspectives and future directions. Curr Opin Ophthalmol 2012; 23(02):96-104

5 Fellman RL, Mattox C, Singh K, et al. American Glaucoma Society Position Paper: microinvasive glaucoma surgery. Ophthalmol Glaucoma 2020;3(01):1-6
6 Accreditation Council for Graduate Medical Education. Required minimum number of procedures for graduating residents in ophthalmology. Available at: https://www.acgme.org/Portals/ 0/PFAssets/ProgramResources/240_Oph_Minimum_Numbers. pdf. Published 2013. Accessed May 15, 2020

7 Shah M. Micro-invasive glaucoma surgery-an interventional glaucoma revolution. Eye Vis (Lond) 2019;6(01):29

8 Arora KS, Robin AL, Corcoran KJ, Corcoran SL, Ramulu PY. Use of various glaucoma surgeries and procedures in medicare beneficiaries from 1994 to 2012. Ophthalmology 2015;122(08): $1615-1624$

9 Chadha N, Warren JL, Liu J, Tsai JC, Teng CC. Seven- and eight-year trends in resident and fellow glaucoma surgical experience. Clin Ophthalmol 2019;13:303-309

10 Accreditation Council for Graduate Medical Education. Ophthalmology Case Logs National. Data Report. Accessed on May 15, 2020 at: https://acgme.org/Portals/0/Opthalmology_National_ Report_Program_Version.pdf2019

11 Reardon W, Sharpe RA, Sharpe E. Retrospective analysis of resident-performed microinvasive glaucoma surgery with the iStent. Invest Ophthalmol Vis Sci 2015;56(07):2694

12 Binenbaum G, Volpe NJ. Ophthalmology resident surgical competency: a national survey. Ophthalmology 2006;113(07): 1237-1244 